\title{
Estudo citofotométrico da expressão dos marcadores tumorais Ki- 67 e CD34 no adenocarcinoma de próstata
}

\author{
Cytophotometric expression of tumor antigen markers Ki-67 and CD-34 in \\ prostate adenocarcinoma
}

Paulo Faria Barbosa ${ }^{1}$; Osvaldo Malafaia, ECBC-PR²; Jurandir Marcondes Ribas-Filho, TCBC-PR 2; Nicolau Gregori Czeczko, TCBC-PR²; Carmen Marcondes Ribas ${ }^{2}$; Ronaldo Mafia Cuenca, TCBC-DF²; Domingos Candiota Chula ${ }^{1}$

\section{R E S U M O}

\begin{abstract}
Objetivo: Quantificar a porcentagem da imunomarcação no índice de marcagem e densidade óptica do Ki-67 e CD34 no adenocarcinoma de próstata e compará-las entre si. Métodos: Foram estudados, através de imunoistoquímica, o Ki-67 e o CD34 em 34 casos de adenocarcinoma de próstata provenientes de prostatectomia radical no período de 2000 a 2005 realizado no Hospital Regional do Gama em Brasília. Estes marcadores foram quantificados através do software SAMBA 4000 ® Sistema de Análise Microscópica de Busca Automática e do software IMMUNO® para análise das variáveis índice de marcagem e densidade óptica. Para avaliação da associação entre as expressões do marcador, foi estimado o coeficiente de correlação de Spearman. Para a comparação do tipo de lesão, foi usado o teste t de Student em amostras pareadas e não paramétrico de Wilcoxon. Resultados: Dos 34 blocos que foram para leitura dos marcadores tumorais, 15 marcaram expressão com Ki-67, 34 com CD34 e 14 com ambos os marcadores. O índice de marcagem do CD34 teve valor mediano de $72,72 \%$, valor mínimo 5,14\% e valor máximo $88,81 \%$. O índice de marcagem do Ki-67 teve mediana de 73,78\%, mínimo de 16,87\% e máximo de 87,47\%. A densidade óptica do CD34 teve mediana de 48,33, mínimo de 35,65 e máximo de 85,86. Na densidade óptica do Ki-67 o valor da mediana foi 40,03 sendo a mínima de 21,53 e a máxima de 52,43. Conclusão: A expressão citofotométrica do Ki-67 teve índice médio de marcação de $64,04 \%$ e o CD34 de 61,64\%. A expressão citofotométrica da densidade óptica média do Ki-67 foi de 39,49 e no CD34 de 53,69. Há diferença significativa entre a imunomarcação do Ki-67 e CD34 em relação à densidade óptica $(p=0,025)$, não havendo diferença significativa no índice de marcagem $(p=0,470)$.
\end{abstract}

Descritores: Marcadores biológicos de tumor. Antígenos CD34. Adenocarcinoma. Neoplasias da próstata. Citometria de imagem. Software.

\section{INTRODUÇÃO}

O adenocarcinoma da próstata é um dos tumores mais frequentes e a $11^{\text {a }}$ causa de morte nos homens, com estimativa de 9.000 óbitos ao ano. Está entre os de melhor prognóstico quando diagnosticado precocemente. Ele surge porque as múltiplas divisões celulares que ocorrem com o passar dos anos se acompanham de discreta fragmentação dos cromossomos que se privam de parte do seu material genético. Com o decorrer do tempo, acumulam-se perdas dos genes supressores (p53, o Rb e o p21), que liberam a atividade dos proto-oncogenes e permitem a degeneração das células prostáticas. A morte celular programada pela apoptose e a angiogênese teem forte relação com a intensidade da carcinogênese.

A expressão da proteína humana Ki-67 é estreitamente associada com a proliferação celular. O fato de estar presente durante todas as fases ativas do ciclo celular (G1,
S, G2 e mitose) e não estar nas células em repouso (G0), faz do Ki-67 excelente marcador para determinar a fração estimulante do crescimento de conhecida população celular' ${ }^{1}$.

O marcador de células hematopoiéticas primitivas e endoteliais CD34, é marcador que modula a formação de novos vasos ou angiogênese, e tem sido avaliado nos cânceres prostáticos através da imunoistoquímica em vários trabalhos ${ }^{2,3}$.

A análise da imagem nuclear das células tumorais, com o incremento da informática na área médi$\mathrm{ca}$, tem sido destacada pela citofotometria de imagem e contribuido na avaliação diagnóstica das neoplasias que acometem o ser humano ${ }^{4}$. Neste tipo de análise simultaneamente obtém-se a informação microscópica e matemática; as imagens analógicas observadas na microscopia são transformadas em imagens numéricas quando digitalizadas e, assim, é possível o tratamento estatístico dos dados digitalizados em pontos de imagem (pixels).

\footnotetext{
Trabalho realizado pelo Programa de Pós-Graduação em Princípios da Cirurgia e Instituto de Pesquisas Médicas da Faculdade Evangélica do Paraná/Hospital Universitário Evangélico de Curitiba, Curitiba, PR, Brasil.

1. Mestre do Programa de Pós-Graduação em Princípios Da Cirurgia e Instituto de Pesquisas Médicas da Faculdade Evangélica do Paraná/Hospital Universitário Evangélico de Curitiba, Curitiba, PR-BR. 2. Doutor, Professor Permanente do Programa de Pós-Graduação em Princípios da Cirurgia e Instituto de Pesquisas Médicas da Faculdade Evangélica do Paraná/Hospital Universitário Evangélico de Curitiba, Curitiba, PR-BR.
} 
Este estudo no adenocarcinoma de próstata tem por objetivos: a) quantificar a expressão citofotométrica do índice de marcagem do Ki-67 e CD34; b) quantificar a densidade óptica média da imunomarcação do Ki-67 e CD34; c) comparar a imunomarcação dos dois marcadores entre si.

\section{MÉTODOS}

Este trabalho foi realizado no Instituto de Pesquisas Medicas (IPEM) e no Laboratório de Citologia e Histologia Ltda - CITOLAB, em Curitiba/Paraná tendo sido avaliado e aprovado pela Comissão de Ética em Pesquisa da Sociedade Evangélica Beneficente de Curitiba.

Foram selecionados 50 blocos de parafina de adenocarcinoma de próstata, sendo possível, deste montante, realizar 38 lâminas. Destas, 34 tiveram campos lidos, sendo 15 com Ki-67 e 34 com CD34. Em 14 lâminas, ambos os marcadores foram lidos.

Todos os tumores estavam identificados e processados de acordo com técnicas histológicas de rotina, tendo sido fixados em solução de formol, tamponados e emblocados em parafina.

As coletas foram obtidas no Serviço de Anatomia Patológica do Hospital Regional do Gama - DF, no período de 2000 a 2005. As amostras que constituíram a casuística deste estudo foram selecionadas entre aquelas que apresentaram confirmação diagnóstica através de critérios histológicos de adenocarcinoma de próstata, e estavam adequadas para a realização da imunomarcagem.

Utilizou-se o sistema SAMBA 4000® (Sistema de Análise Microscópica de Busca Automática), desenvolvido pela Alcatel (Grenoble - França) para leitura das lâminas e software IMMUNO® 4.0. Este sistema é constituído por hardware capaz de captar imagens microscópicas e software que interpreta as imagens captadas.

Neste estudo utilizou-se o antígeno Ki-67 antihumano (monoclonal de camundongo), clone MIB-1, código $\mathrm{N}^{\circ} \mathrm{M} 7240$.

Utilizou-se também o antígeno CD34 (marcador de células endoteliais) Ab-1 (anticorpo monoclonal de rato), clone QEBnd/10.

Para estudo da frequência do índice de marcagem do Ki-67 e do CD34, as lâminas foram lidas em porcentagem e divididas com índices em escala de: 0 a 20; 20,01 a 40; 40,01 a 60; 60,01 a 80; 80,01 a 100.

Para a frequência da densidade óptica do Ki-67 as lâminas foram divididas em amostras com densidade de: 20,01 a 30; 30,01 a 40; 40,01 a 50; 50,01 a 60. Foram lidas em pixels.

Para a frequência da densidade óptica do CD34 as lâminas foram divididas em amostras com densidade de: 30,01 a 40; 40,01 a 50; 50,01 a 60; 60,01 a 70; 70,01 a 80; 80,01 a 90 e também lidas em pixels.

Os resultados obtidos foram expressos por médias, medianas, valores mínimos, valores máximos e desviospadrão. A condição de normalidade das variáveis quantitativas foi investigada usando-se o teste de Shapiro-Wilks. Para avaliar o grau de associação entre a expressão dos marcadores, foi estimado o coeficiente de correlação de Pearson ou de Spearman. A comparação dos marcadores foi feita usando-se o teste $t$ de Student para amostras pareadas ou o teste não-paramétrico de Wilcoxon quando apropriado.

\section{RESULTADOS}

De 34 blocos aproveitados para estudo imunoistoquímico, 15 casos expressaram o Ki-67 (44,11\%) e 34 (100\%) o CD34; destes, 14 marcaram expressão em ambos marcadores.

\section{Indice de marcagem e densidade óptica do $\mathrm{KI}-67$ e CD34}

Na tabela 1 são apresentados os números de casos que marcaram para o Ki-67 e CD34 nas médias, medianas, valores mínimos, valores máximos e desviospadrão do índice de marcagem e densidade óptica, considerando-se todas as lâminas que foram lidas. A média do índice de marcagem do Ki-67 (63,82\%) foi mais expressiva que a do CD34 (51,97\%); a densidade óptica do CD34 foi mais expressiva que a do Ki-67.

\section{Distribuição da frequência do índice de marcagem do $\mathrm{Ki}-67$}

Na figura 1 são demonstrados os índices de marcagem da expressão do marcador Ki-67, divididos em cinco grupos na seguinte maneira: 0 a 20, uma lâmina lida $(6,67 \%) ;$ de 20,01 a 40, 1 lâmina lida (6,67\%); 40,01 a 60 , duas lâminas lidas, (13,33\%); 60,01 a 80, nove lâminas lidas (60,00\%); 80,01 a 100, duas lâminas lidas $(13,33 \%)$

Testou-se a hipótese nula de que os dados teem distribuição normal versus a hipótese alternativa de que eles não a teem. Os resultados indicaram que, para o índice de marcagem do Ki-67, não há normalidade dos casos.

Tabela 1 - Índice de marcagem e densidade óptica do Ki-67 e CD34.

\begin{tabular}{lcccccc}
\hline Variável & N & Média & Mediana & Mínimo & Máximo & Desvio-padrão \\
\hline Índice de marcagem do CD34 & 34 & 52,80 & 71,21 & 2,90 & 88,81 & 31,94 \\
Densidade óptica do CD34 & 34 & 51,97 & 48,03 & 31,85 & 85,86 & 13,28 \\
Índice de marcagem do Ki-67 & 15 & 63,82 & 72,62 & 16,87 & 87,47 & 21,41 \\
Densidade óptica do Ki-67 & 15 & 39,39 & 38,12 & 21,53 & 52,43 & 9,30 \\
\hline
\end{tabular}


Na figura 2 são apresentadas as distribuições de frequências da densidade óptica da expressão do Ki-67, divididas em quatro grupos, na seguinte maneira: 20,01 a 30, 2 lâminas lidas (13,33\%); 30,01 a 40, 6 lâminas lidas (40,00\%); 40,01 a 50, 5 lâminas lidas (33,33\%); 50,01 a 60, 2 lâminas lidas(13,33\%).

Testou-se a hipótese nula de que os dados teem distribuição normal, versus a hipótese alternativa de que não a teem. Os resultados indicaram que não houve rejeição da hipótese de normalidade dos casos.

\section{marcagem do CD34 \\ Distribuição da frequência do índice de}

Na figura 3 são apresentadas as distribuições de frequências do índice de marcagem do CD34, divididos em cinco grupos na seguinte maneira: 0 a 20, oito lâminas lidas $(23,53 \%) ;$ de 20,01 a 40 , cinco lâminas lidas $(14,71 \%) ; 40,01$ a 60 , uma lâmina lida $(2,94 \%) ; 60,01$ a 80, 13 lâminas lidas (38,24\%); 80,01 a 100, sete lâminas lidas (20,59\%).

Testou-se a hipótese nula de que os dados tenham distribuição normal, versus a hipótese alternativa de que não a possuem. Os resultados dos testes indicaram que não há normalidade da frequência dos casos.

$\mathrm{Na}$ figura 4 são apresentadas as distribuições das frequências do índice de densidade óptica divididos em seis

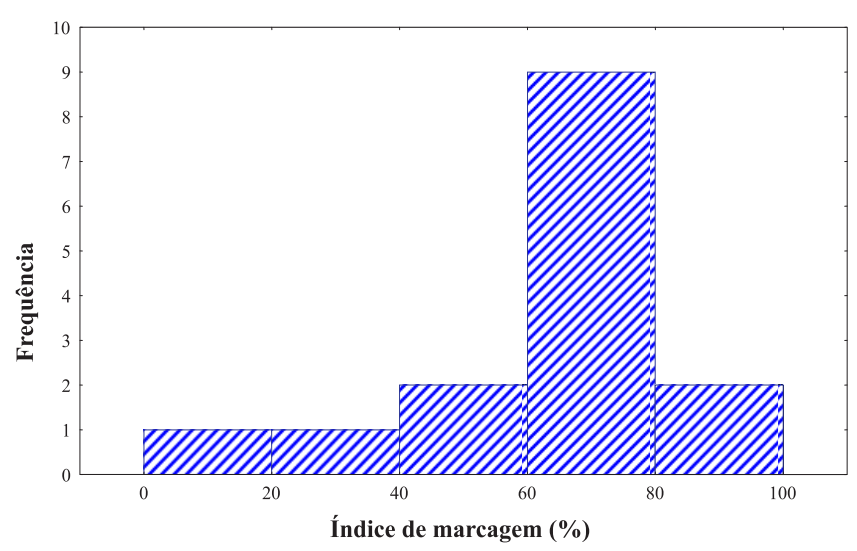

Figura 1 - Índice de marcagem do Ki-67.

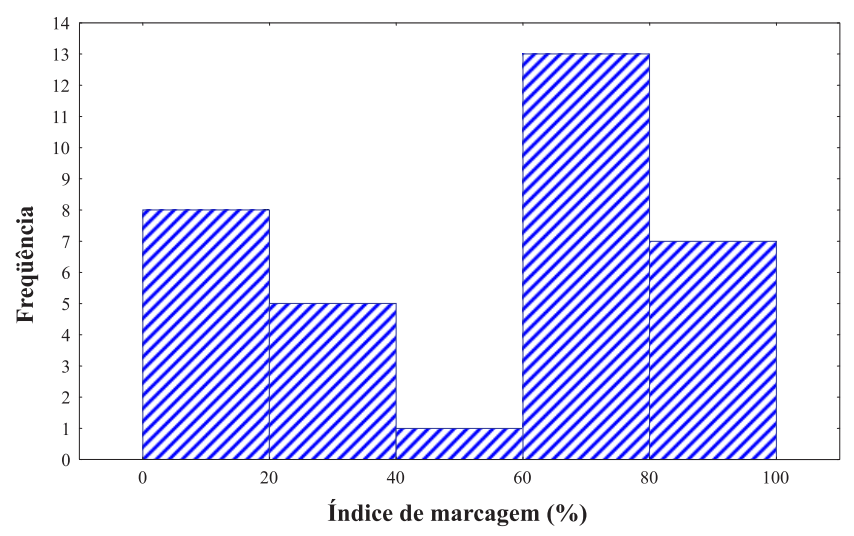

Figura 3 - Índice de marcagem do CD34. grupos na seguinte maneira: 30,01 a 40, oito lâminas lidas (23,53\%); de 40,01 a 50, 11 lâminas lidas (32,35\%); 50,01 a 60 , seis lâminas lidas $(17,65 \%) ; 60,01$ a 70 , seis lâminas lidas (17,65\%); 70,01 a 80, uma lâmina lida (2,94\%); de 80,01 a 90, duas lâminas lidas (5,88\%).

Testou-se a hipótese nula de que os dados têm distribuição normal, versus a hipótese alternativa de que não. Os resultados indicaram que não houve rejeição de normalidade dos casos.

\section{Comparação entre os marcadores}

Na figura 5 mostra-se a comparação dos resultados do índice de marcagem do Ki-67 com os do CD34, com seus valores mínimo, máximo e de mediana, nas 14 lâminas lidas por ambos os marcadores.

O resultado do teste indicou a não rejeição da hipótese nula $(p=0,470)$. Sendo assim, não se pode afirmar que existe diferença significativa entre Ki-67 e CD34 em relação ao índice de marcagem.

\section{CD34}

\section{Densidade óptica dos marcadores Ki-67 e}

Na figura 6 estão apresentados o desvio-padrão, o erro-padrão e a média da densidade óptica de ambos marcadores. Pode-se afirmar que existe diferença significativa entre eles em relação à média da densidade óptica.

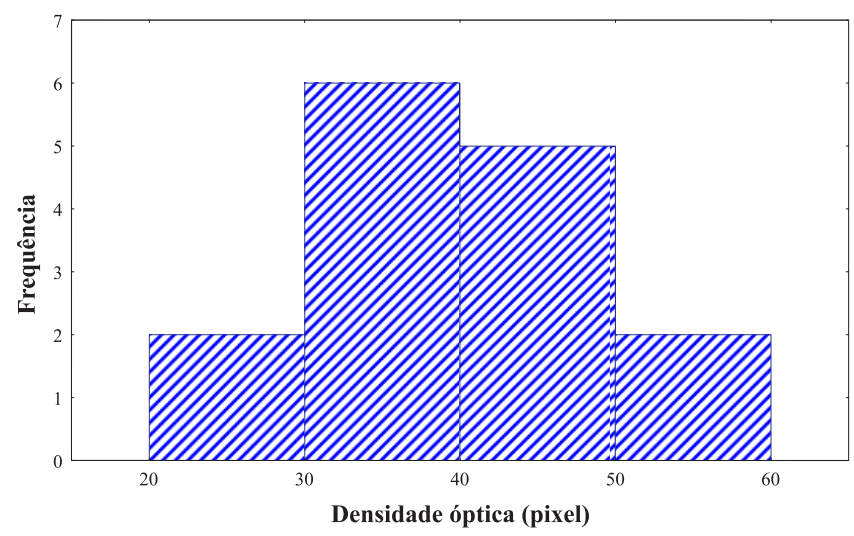

Figura 2 - Densidade óptica do Ki-67.

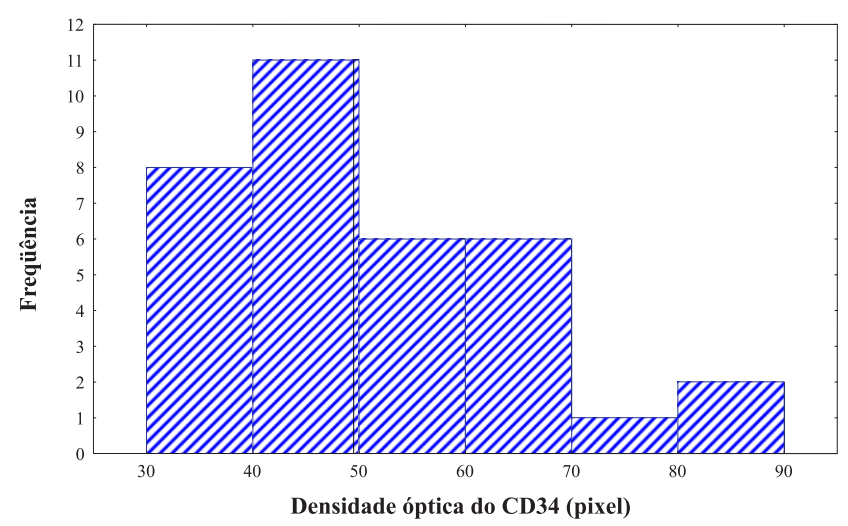

Figura 4 - Densidade óptica do CD34. 


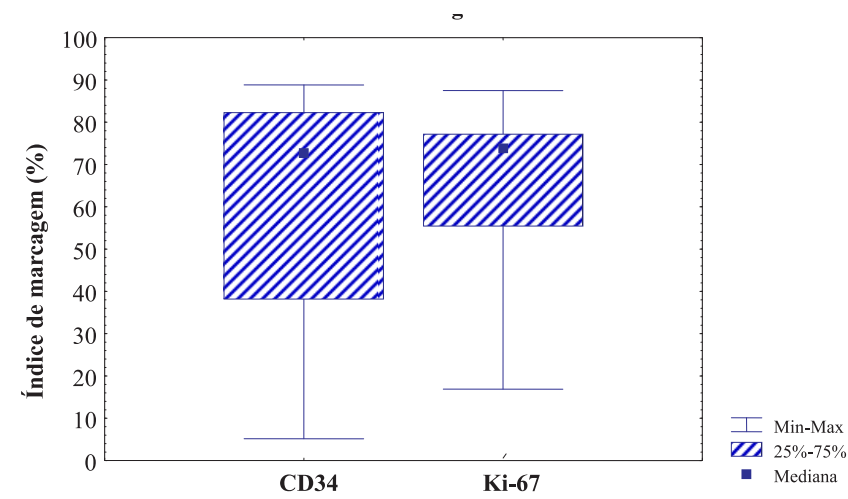

Figura 5 - Diferença do índice de marcagem do Ki-67 e CD34.

Observou-se que a média da densidade óptica do CD34 é maior que a do Ki-67.

\section{DISCUSSÃO}

Na história natural do adenocarcinoma de próstata, o primeiro evento que ocorre é a proliferação irregular de células. Em relação a isso, acredita-se que quanto maior a atividade proliferativa do tumor, pior será seu comportamento biológico. Como consequência, muito esforço tem sido feito no desenvolvimento de métodos objetivos para avaliar a proliferação celular e estudar o significado destes métodos no estudo das neoplasias, especialmente no seu prognóstico.

Nos métodos imunoistoquímicos, características técnicas e aspectos metodológicos são frequentemente responsáveis pela variação dos resultados. Neste estudo, foram analisados os índices de marcagem e densidade óptica dos marcadores tumorais Ki-67 e CD34. Em que pese a facilidade encontrada para conseguir o material pesquisado de 50 blocos de parafina contendo tumor de próstata, só foram lidos as expressões citofotométricas em 34 lâminas, devido a qualidade da conservação do material não estar adequada. A não-observância rigorosa de protocolos de parafinização e de estocagem - locais que não atentem a critérios rígidos de cuidados com exposição de luz, temperatura e umidade relativa do ar -, foram fatores decisivos para a queda da qualidade e quantidade da amostragem inicial dos blocos.

Os marcadores biológicos tumorais relacionamse diretamente com as transformações bioquímicas, histológicas e morfológicas em todo o organismo. O seu uso está se convertendo em importante ferramenta para examinar os níveis de transformação celular e molecular, especialmente quando se trata de ácidos nucléicos e proteínas. São especialmente empregados para medir a exposição a determinados agentes, detectar a severidade de alguma resposta tóxica e predizer possíveis resultados 5 . 0 Ki-67 é bom marcador de proliferação celular. Ele marca uma proteína presente em todas as fases de vida celular menos na fase $\mathrm{G} 0$, tendo relação direta com a fração de crescimento de uma população celular ${ }^{1}$. Neste trabalho

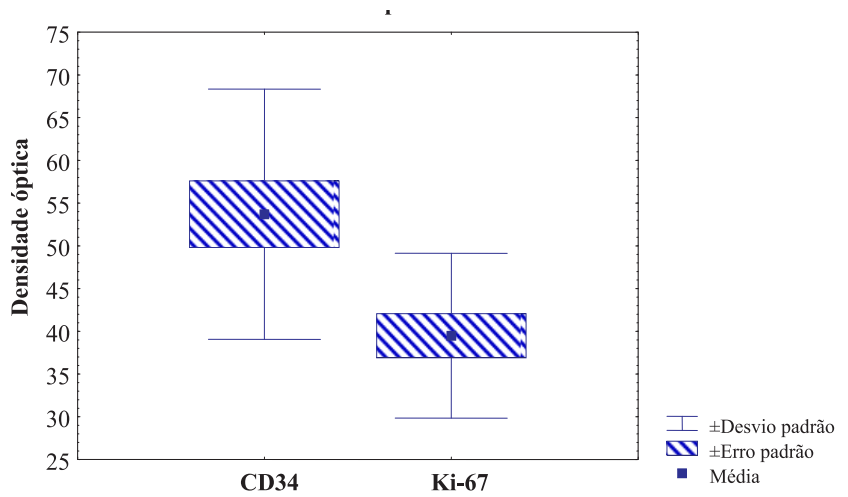

Figura 6 - Diferença da densidade óptica do Ki-67 e CD34.

pode-se avaliar que não existe diferença significativa no índice de marcagem do Ki-67 e CD34, pois a média de ambos os marcadores foram respectivamente $61.64 \%$ (CD34) e 64.04\% (Ki-67). No presente estudo a leitura com o marcador CD34 foi possível em 34 lâminas que representaram porcentagem de positividade de 100\%.

A expressão da proteína Ki-67 é requisito absoluto para progressão através do ciclo de divisão celular. 0 fato de estar presente durante todas as fases ativas do ciclo celular (G1, S, G2 mitose) e não estar nas células em repouso (G0), faz do Ki-67 um bom marcador para determinar a fração estimulante do crescimento de uma conhecida população celular. Isto determina que esteja bem caracterizada no nível molecular, sendo usada como marcador de proliferação celular, porém sua significância funcional ainda não está bem esclarecida1.

Desde que se observou que a fração de crescimento é somente um dos fatores que influenciam o comportamento tumoral, seria ingênuo acreditar que essa medida seria um parâmetro isolado para fornecer ao clínico, com exatidão, o prognóstico definitivo para todos os tumores. Das 34 lâminas pesquisadas de adenocarcinoma de próstata, apenas 15 tiveram positividade para o Ki-67, perfazendo um total de 44,11\%. Neste trabalho, o Ki-67 não teve índice de positividade em todas as lâminas lidas mesmo sendo todas elas de adenocarcinoma de próstata.

Mucci, et al. ${ }^{6}$, verificaram em 45 pacientes submetidos à prostatectomia radical se o Ki-67 apresentava níveis de expressão significativamente diferentes em tecidos de próstata normal, no alto grau de neoplasia intraepitelial e no adenocarcinoma de próstata. Constataram que havia aumentos estatisticamente significativos na expressão do Ki-67 do tecido normal em relação à neoplasia intra-epitelial e ao adenocarcinoma.

Neste estudo o Ki-67 teve percentagem de positividade em 15 lâminas do total de 34 estudadas. Outros autores como Rioux-Leclerc, et al. ${ }^{7}$, verificaram a utilidade da expressão do Ki-67 no diagnóstico diferencial da neoplasia intra-epitelial prostática e o adenocarcinoma de próstata, comparando o índice de marcação de Ki-67 em 17 casos de cada doença. A atividade proliferativa foi estatisticamente mais alta no adenocarcinoma (32\%) em relação à neoplasia intra-epitelial prostática (6\%). 
Os marcadores tumorais devem ser utilizados de forma conjunta para que as diferentes vias de ativação possam ser acessadas e novos alvos descobertos. Esses novos alvos irão auxiliar no diagnóstico, prognóstico e desenvolvimento de tratamentos e drogas anti-câncer no futuro.

O antígeno CD34 teve porcentagem de positividade na marcagem em 34 lâminas perfazendo um total de $100 \%$ da amostra selecionada para o estudo, confirmando os achados de outros autores ${ }^{8,9}$, confirmando a presença de angiogênese em todo o material estudado.

Existem diferentes anticorpos antiendoteliais e diferentes maneiras de avaliar as densidades microvasculares. Offersen, et al. ${ }^{10}$,examinaram dois anticorpos antiendoteliais (anti-CD34 e antifator de Von Willebrand) e dois métodos de medir a angiogênese em cânceres de próstata: densidade de microvasos e Chalkley. Ambos os métodos foram avaliados com o anticorpo antiCD34. Para a contagem dos microvasos, neste trabalho, empregou-se método computadorizado, com o software IMMUNO® do Sistema SAMBA, e não a contagem direta subjetiva, metodologia usada tanto por Bono, et al. ${ }^{11}$, como por Offersen, et al. ${ }^{10}$.

O conhecimento da topografia da microvasculatura tem implicações na quantificação da angiogênese nos espécimes obtidos por biópsias com aguIha12, o que não interfere nos casos do presente estudo, em função de as amostras terem sido obtidas de prostatectomia radical. Bettencourt, et al. ${ }^{9}$, afirmaram que a medida quantitativa da angiogênese pode ser realizada pela densidade de microvasos, determinando a expressão dos antígenos endoteliais. Na presente pesquisa, em conformidade com o achado desses autores, utilizou-se o marcador CD34, obtendo-se percentagem de positividade em todas as 34 lâminas avaliadas.

No adenocarcinoma de próstata: a) a expressão citofotométrica teve índice médio de marcagem de 64,04\% com Ki-67 e de 61,64\% com o CD34 ; b) a mesma, em relação à densidade óptica, mostrou média de 39,49 com o Ki-67 e de 53,69 com o (D34; c) há diferença significativa entre a imunomarcação do Ki-67 e CD34 em relação a densidade óptica $(p=0,025)$, não havendo no índice de marcagem $(p=0,470)$.

\title{
A
}

\begin{abstract}
Objective: To quantify the percentage of immunostaining through the labeling index as well as the optical density of Ki-67 and CD34 in prostate adenocarcinoma and compare the results between markers. Methods: Markers Ki-67 and CD34 were studied using immunohistochemistry in 34 cases of prostate adenocarcinoma from radical prostatectomies performed at the Hospital Regional do Gama in Brasilia, Brazil from 2000 through 2005. Those markers were quantified using the SAMBA 4000® software Automated Scanning Microscopic Analysis System - and the IMMUNO® software in the analysis of the variables labeling index and optical density. Spearman's correlation coefficient was estimated in order to evaluate the association between the expression levels of the markers. For the comparison of lesion types, Student's paired t-test and the nonparametric Wilcoxon test were used. Results: Of the 34 blocks referred for the study of the tumor markers, 15 were positive for Ki-67, 34 showed CD34 expression, and 14 were positive for both markers. The median value for the labeling index of CD34 was $72.72 \%$; the minimum was $5.14 \%$ and the maximum, $88.81 \%$. The median for the Ki-67 labeling index was $73.78 \%$, while the minimum was $16.87 \%$, and the maximum, $87.47 \%$. The median value for the optical density of CD34 was 48.33, the minimum was 35.65 and the maximum, 85.86. For the optical density of Ki-67, the median was 40.03, while the minimum and maximum values were 21.53 and 52.43 , respectively. Conclusion: The cytophotometric expression of Ki-67 had a mean labeling index of 64.04\%, and the mean CD34 labeling index was $61.64 \%$. The cytophotometric expression of the mean optical density of Ki-67 was 39.49, while for CD34 it was 53.69. There was a significant difference between Ki-67 and CD34 immunostaining with respect to optical density $(P=0.025)$; no significant difference occurred regarding labeling index $(P=0.470)$.
\end{abstract}

Key words: Tumor markers, biological. Antigens, CD34. adenocarcinoma. Prostatic neoplasms. Image cytometry. Software.

\section{REFERÊNCIAS}

1. Scholzen T, Gerdes J. The Ki-67 protein: from the known and the unknown. J Cell Physiol. 2000; 182(3):311-22.

2. Banerjee AG, Liu J, Yuan Y, Gopalakrishnan VK, Johansson SL, Dinda AK et al. Expression of biomarkers modulating prostate cancer angiogenesis: differential expression of annexin II in prostate carcinomas from India and USA. Mol Cancer. 2003; 2:34.

3. Lutsenko SV, Kiselev SM, Severin SE. Molecular mechanisms of tumor angiogenesis. Biochemistry (Mosc). 2003; 68(3):286-300.

4. Lima EB. Estudo citofotométrico do ADN de câncer gástrico [dissertação]. Curitiba (PR): Universidade Federal do Paraná; 2000.

5. Abu-Qare AW, Abou-Donia MB. Biomarkers of apoptosis: release of cytochrome c, activation of caspase-3, induction of 8-hydroxy- 2'-deoxyguanosine, increased 3-nitrotyrosine, and alteration of p53 gene. J Toxicol Environ Health B Crit Rev. 2001; 4(3):313-32.

6. Mucci NR, Rubin MA, Strawderman MS, Montie JE, Smith DC, Pienta KJ. Expression of nuclear antigen Ki-67 in prostate cancer needle biopsy and radical prostatectomy specimens. J Natl Cancer Inst. 2000; 92(23):1941-2.

7. Rioux-Leclerc N, Leray E, Pattard JJ, Lobel B, Guille F, Joun F et al. The utility of Ki-67 expression in the differenctial diagnosis of prostatic intraepitelial neoplasia and ductal adenocarcinomas. Human Pathol. 2005; 36(5):531-5.

8. Hall MC, Troncoso P, Pollack A, Zhau HY, Zagars GK, Chung LW, von Eschenbach AC. Significance of tumor angiogenesis in clinically localized prostate carcinoma treated with external beam radiotherapy. Urology. 1994; 44(6):869-75. 
9. Bettencourt MC, Bauer JJ, Sesterhenn IA, Connelly RR, Moul JW. CD34 immunohistochemical assessment of angiogenesis as a prognostic marker for prostate cancer recurrence after radical prostatectomy. J Urol. 1998; 160(2):459-65.

10. Offersen BV, Borre M, Sørensen FB, Overgaard J. Comparison of methods of microvascular staining and quantification in prostate carcinoma: relevance to prognosis. APMIS. 2002; 110(2):177-85.

11. Bono AV, Celato N, Cova V, Salvadore M, Chinetti S, Novario R. Microvessel density in prostate carcinoma. Prostate Cancer Prostatic Dis. 2002; 5(2):123-7.

12. Silberman MA, Partin AW, Veltri RW, Epstein Jl. Tumor angiogenesis correlates with progression after radical prostatectomy but not with pathologic stage in Gleason sum 5 to 7 adenocarcinoma of the prostate. Cancer. 1997; 79(4):772-9.
Recebido em 10/12/2008

Aceito para publicação em 20/02/2009

Conflito de interesse: nenhum

Fonte de financiamento: nenhuma

\section{Como citar este artigo:}

Barbosa PF, Malafaia O, Ribas-Filho JM, Czeczko NG, Ribas CM, Cuenca RM, Chula DC. Estudo citofotométrico da expressão dos marcadores tumorais Ki- 67 e CD34 no adenocarcinoma de próstata. Rev Col Bras Cir. [periódico na Internet] 2009; 36(6). Disponível em URL: http:// www.scielo.br/rcbc

\section{Endereço para correspondência:}

Paulo Faria Barbosa

E-mail: Ipem@evangelico.org.br 